\title{
Stochastic Background of Gravitational Waves Generated by Compact Binary Systems
}

\section{Edgard F. D. Evangelista}

National Institute for Space Research - Astrophysics Division, São José dos Campos, Brazil

E-mail: edgard.inpe@gmail.com

\section{José C. N. de Araujo}

National Institute for Space Research - Astrophysics Division, São José dos Campos, Brazil

E-mail: jcarlos@das.inpe.br

Binary Systems are the most studied sources of gravitational radiation, both theoretically and observationally. Theoretically, the mechanisms of emission and the behaviour of the orbital parameters are well known and can be written in analytic form in several cases, which represents a great advantage in terms of computational work; observationally, even though gravitational waves have not been detected yet, the strongest indication of its existence have arisen from the observation of binary systems, more specifically the Hulse-Taylor binary pulsar. When one begins to detect gravitational radiation, one of the observed pattern of the signals will probably be of stochastic background nature because there will be in some cases a superposition of the signals emitted by a plenty of sources around the Universe. So, our task here is to develop a method of summing the gravitational waves emitted by cosmological binary compact systems in order to find out the shape of the spectra, that is, we will develop a method for obtaining the stochastic background generated by these systems. We use here an analogy with a problem of statistical mechanics in order to perform this sum, besides taking into account the temporal variation of the orbital parameters of the systems.

25th Texas Symposium on Relativistic Astrophysics - TEXAS 2010

December 06-10, 2010

Heidelberg, Germany 


\section{Introduction}

Binary systems are well studied sources of gravitational radiation. Particularly, we are interested in systems that are formed by neutron stars and black holes, which in general emit gravitational waves in higher amplitudes than the other types of binaries and, by this reason, the probability of detection could be increased. Besides, if we consider that the Universe is filled with a large population of compact binary systems emitting gravitational waves, we may suppose that in many cases the superposition of the signals will generate a smooth spectrum of characteristic shape and of stochastic type. So, the aim of this paper is to present a method for the calculation of the stochastic background generated by compact binary systems in circular orbits. First we will present a brief description of the theory concerning the binary systems and their emitted waves; after we will present a method for the calculation of the background and, finally, we will show some results.

\section{Power Emitted in Gravitational Waves}

The total luminosity of gravitational radiation emitted by a binary system is given by

$$
\frac{d E}{d t}=\frac{32 G^{7 / 3}}{5 c^{5}} \frac{\left(m_{1} m_{2}\right)^{2}}{\left(m_{1}+m_{2}\right)^{2 / 3}} \omega^{10 / 3} F(e)
$$

where $m_{1}$ and $m_{2}$ are the masses of the components of the system and $\omega$ is the angular orbital frequency. We can separate the total power in a sum of terms, where each one of them correspond to one harmonic:

$$
F(e)=\sum_{n=1}^{\infty} g(n, e)
$$

where $g(n, e)$ are very complicated functions of the harmonic number $n$ and the eccentricity $e$ and may be found in [1]. In the present case, we shall restrict ourselves to circular orbits, where $F(e)=1$, and deduce an expression for the temporal variation of the orbital frequency of the system. So, we may use the chain rule

$$
\frac{d \omega}{d t}=\frac{d \omega}{d r} \frac{d r}{d E} \frac{d E}{d t}
$$

and get

$$
\frac{d \omega}{d t}=\frac{96 m_{1} m_{2}}{5 c^{5}} G^{5 / 3}\left(m_{1}+m_{2}\right)^{-1 / 3} \omega^{11 / 3}
$$

Solving this differential equation, we have

$$
\omega^{-8 / 3}=\omega_{0}^{-8 / 3}-\frac{8}{3} K\left(t-t_{0}\right)
$$

where

$$
K=\frac{96 m_{1} m_{2}}{5 c^{5}} G^{5 / 3}\left(m_{1}+m_{2}\right)^{-1 / 3}
$$

Moreover, we may write the angular frequency $\omega$ in therms of the orbital frequency $\Omega$ :

$$
\omega=2 \pi \Omega
$$




\section{Distribution Functions}

Binary systems have different values for the orbital parameters such as eccentricity and semimajor axis, then we can use distribution functions to describe them. An example of such a function may be found in [2] and is given by:

$$
F(a, e) \text { dade }=\frac{3}{2} \frac{a^{1 / 2}}{a_{\max }^{3 / 2}} e\left(1-e^{2}\right)^{-3 / 2} d a d e
$$

here, $a$ is the semi-major axis, $e$ is the eccentricity and $a_{\max }$ is the maximum orbital distance observed in a given population of systems. Besides, we can obtain separate distribution for the $a$ and $e$, say $F(a)$ and $F(e)$, by performing convenient integration over $F(a, e)$. More specifically, in order to obtain $F(a)$ we integrate over $e$, and the same is valid to $F(e)$. So we have to $F(a)$ :

$$
F(a) d a=\frac{3}{2}\left[\left(\frac{a}{a_{\max }}\right)^{3 / 4}-\left(\frac{a}{a_{\max }}\right)^{3 / 2}\right] \frac{d a}{a}
$$

We can use the Kepler Third Law and write Equation 3.2 in terms of the orbital frequency $\Omega$ :

$$
F(\Omega)=\left[\left(\frac{\Omega_{\min }}{\Omega}\right)^{1 / 2}-\frac{\Omega_{\min }}{\Omega}\right] \frac{1}{\Omega}
$$

where $\Omega_{\min }$ is the minimum value of $\Omega$. But the orbital frequency changes, according to Equation 2.4. So, we have to change Equation 3.3 in order to include the temporal dependence. Using Equation 2.4, we have after some algebra:

$$
F\left(\Omega, t, t_{0}\right)=\left(\Omega_{\min }^{1 / 2} \Omega_{0}^{13 / 6}-\Omega_{\min } \Omega_{0}^{5 / 3}\right) \Omega^{-11 / 3}
$$

where we identified the frequency in Equation 3.3 with the initial frequency $\Omega_{0}$ in Equation 2.4. It is worth mentioning that for binary systems in circular orbits the gravitational radiation is of quadrupolar pattern, where the frequency of the emitted waves $v$ is related to $\Omega$ by:

$$
v=2 \Omega
$$

\section{The Stochastic Background Calculation}

In a general way, the stochastic background may be calculate by Equation 4.1, according to [3]:

$$
h_{\mathrm{BG}}^{2}=\frac{1}{v} \int h_{\mathrm{source}}^{2} d R
$$

where $h_{\mathrm{BG}}$ is the amplitude of the spectrum, $h_{\text {source }}$ is the amplitude of a single source, $v$ is the observed frequency and $d R$ is the emission rate of gravitational waves. To binary systems $h_{\text {source }}$ is given by, according to [4] and [10]:

$$
h_{\text {source }}=8.7 \times 10^{-21}\left(\frac{\mu}{M_{\odot}}\right)\left(\frac{M}{M_{\odot}}\right)^{2 / 3}\left(\frac{100 \mathrm{pc}}{d_{\mathrm{L}}}\right)\left(\frac{\mathrm{mHz}}{v}\right)^{-2 / 3}
$$

where $\mu$ is the reduced mass, $M$ is the total mass and $d_{\mathrm{L}}$ is the luminosity distance. So, the main issue is to obtain an expression for $d R$. We are developing a method for this, based on an elementar problem of Statistical Mechanics. 


\subsection{The Statistical Problem}

Let a gas contained in a rectangular box and in thermal equilibrium. An elementar problem is to calculate how many particles (or molecules) of the gas reach a given wall of area $A$ of the box in a time interval, i.e., the aim is to calculate the flux of particles. Acconding to [5], let $d V$ be a differential volume element adjacent to the wall, as showed in Figure 1.

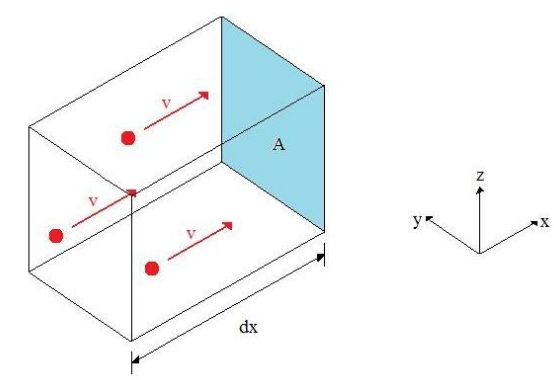

Figure 1: The differential volume element

The volume element is writen as

$$
d V=A v_{x} d t
$$

where all the particles with velocity $v_{x}$ will reach the area $A$ in the time interval $d t$. So, given the total volume of the box and considering a spatially homogeneous distribution of particles, the fraction of particles in that volume $d V$ an with velocities in the interval $d^{3} v$ is given by

$$
d m=\frac{d V}{V} \eta(v) d^{3} v
$$

An integration over velocities (but considering only positive values to the $v_{x}$ because we are considering only particles which are going to the area $A$ ) yields

$$
m=\frac{A d t}{V} \int_{0}^{\infty} d v_{x} \int_{-\infty}^{\infty} d v_{y} \int_{-\infty}^{\infty} d v_{z} v_{x} \eta(v)
$$

In the special case where the box has transversal section $A$, Equation 4.4 becomes

$$
d m=\frac{d x}{L} \eta(v) d^{3} v
$$

In the more general case where the spatial distribution of particles is not constant, the equation above becomes

$$
d m=f(x) \eta(v) d^{3} v d x
$$

where $f(x)$ can be writen as

$$
f(x)=\frac{\varphi(x)}{\int \varphi(x) d x}
$$

where $\varphi(x)$ is the spatial distribution of particles of the gas. Now let us define a new element, which describes the temporal variation of the frequency of the system (note that we use here the frequency of the waves $v$ instead of the orbital frequency $\Omega$ ):

$$
v=\frac{d v}{d t}
$$


Now, let us look at the previous problem and substitute the velocity by $v$ defined above and substitute the spatial coordinate by the frequency $v$. In this case, the frequency distribution $\varphi(v)$ is given by:

$$
\varphi(v)=\varepsilon \int N\left(t_{o}\right) f\left(v, t, t_{o}\right) d t_{o}
$$

where $N\left(t_{o}\right)$ is the binary formation rate by comoving volume element, $f\left(v, t, t_{o}\right)$ represents the fraction of systems at a given instant $t$ and which originated at the instant $t_{o}$ and $\varepsilon$ represents the efficiency of formation of compact binaries in a given population of progenitor stars. Now, let us rewrite Equation 4.7 as

$$
\frac{d R}{d V} \equiv \frac{d n}{d V d t}=\left(\frac{\varphi(v) \frac{d v}{d t}}{\int \varphi(v) d v}\right) \xi(v) d v
$$

Then it is sufficient to integrate over $v$. Besides, it is worth mentioning that there is only one value of $v$ for each value of $v$, as we may conclude by observing Equation 2.4. So, we consider the function $\xi(v)$ as a Dirac's delta multiplied by the total number of systems. A further fact we should consider is that the denominator of the term between parenthesis in Equation 4.11 represents the total number of systems, what we write as $N$. So, performing the integral over $v$, we get

$$
\frac{d R}{d V} \equiv \frac{d n}{d V d t}=\varphi(v) \frac{d v}{d t}
$$

Finally, we write the integral 4.1 as

$$
h_{\mathrm{BG}}^{2}=\frac{1}{V} \int h_{\mathrm{source}}^{2} \frac{d R}{d V} d V
$$

and use Equation 4.12.

Moreover, it is convenient define the strain amplitude, which may be writen as a function of $h_{\mathrm{BG}}$ :

$$
S_{h}=\frac{h_{B G}^{2}}{v}
$$

\section{The spectra}

We show here the calculated spectra, where $S_{h}$ is writen in function of $\varepsilon$ :
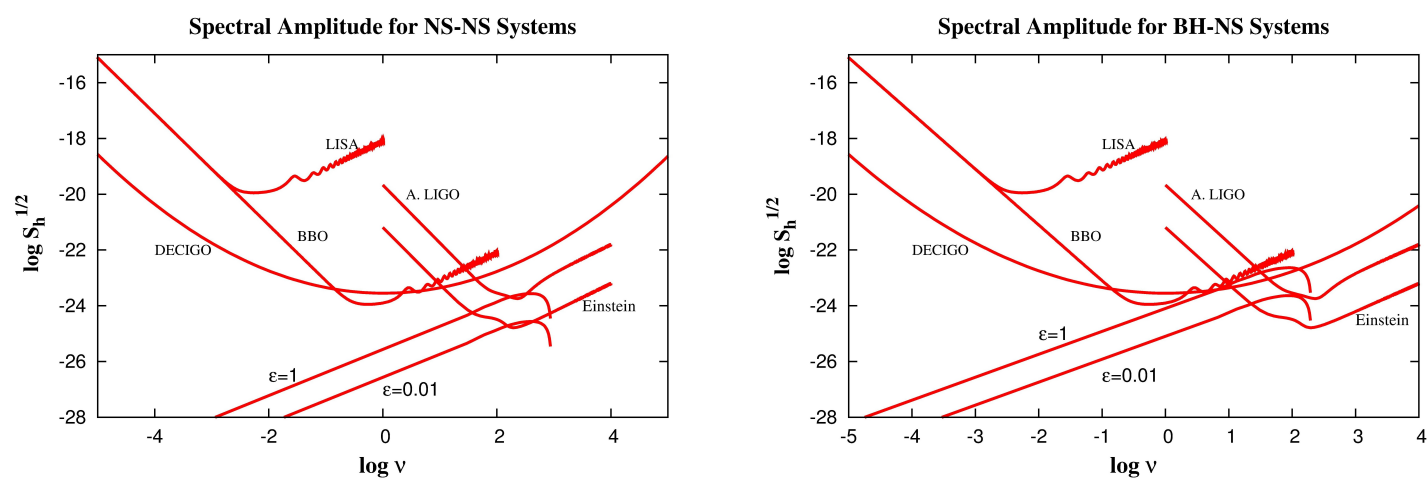


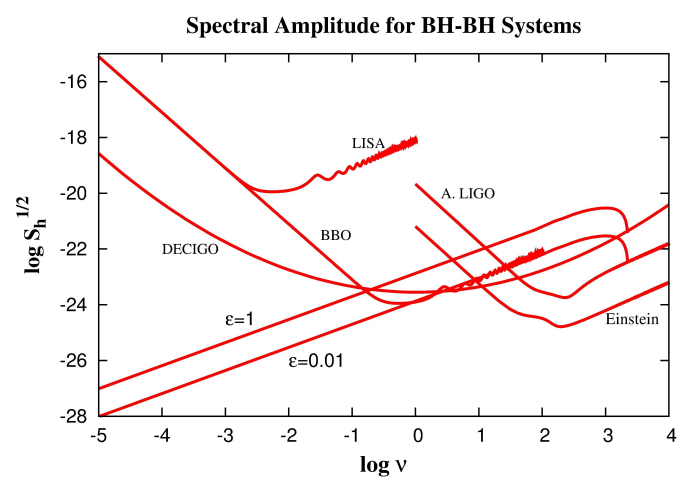

As a general result one conclude that LISA would not be affected by any of the compact binary system noises studied here. Observing the curves for the NS-NS systems, one notes that the background could be in principle be detected by Einstein only if $\varepsilon \sim 1$. BBO, DECIGO and ALIGO would not be affected by such a putative background. BH-NS binaries would not mar the sensitivity of LISA and would only mar ALIGO and marginally mar the DECIGO in the case of $\varepsilon \sim 1$. For more realistic values of $\varepsilon$ the BH-NS would not mar the BBO, DECIGO and ALIGO sensitivity curves. For Einstein, however, only if $\varepsilon \ll 1$ the confusion noise would not be relevant. BH-BH binaries would not be important for BBO and DECIGO only if $\varepsilon \ll 0.01$. For Einstein, however, even for a very small value of $\varepsilon$, say $10^{-6}$, such a binary system would be important.

\section{The Binary Coalescence Rates}

The estimate of the merging rates of binaries is an interesting way of testing the method developed here because there are several known values for these rates and some articles show, in a independent way, results that can be used for comparison (see, e.g., [6], [7], [8] or [9]). In order to calculate the merging rate, we can use our method in a straightforward way: all we need to do is to calculate the rate $d R$, using particular values for the frequency and redshift. For example, in order to calculate the merging rate for a given type of binary system in the neighbourhood of the Galaxy, we calculate $d R$ at the maximum frequency of the gravitational waves emitted by these systems and at redshifts $z \ll 1$. The following table show some results:

\begin{tabular}{|l|l|l|l|}
\hline system & NS-NS & BH-NS & BH-BH \\
\hline merger rate $\left(\mathrm{Mpc}^{-3} \mathrm{yr}^{-1}\right)$ & $\varepsilon 5 \times 10^{-5}$ & $\varepsilon 4 \times 10^{-8}$ & $\varepsilon 6 \times 10^{-4}$ \\
\hline
\end{tabular}

We can note the influence of the efficiency $\varepsilon$ : for higher values of $\varepsilon$ we have higher coalescence rates, and this influence is stronger than in the case of the amplitude $S_{h}$. 


\section{Conclusions}

A new method for the calculation of the stochastic background has been tested for binary systems in circular orbits and the results are promising. In order to submit the method to an additional test we calculated the binary merger rates and compared these values to some present in the literature. In the future we will include the effects of the eccentricity in the calculation of the stochastic background.

\section{Acknowledgments}

The authors would like to thank Brazilian agencies CAPES and FAPESP for financial support and INPE for offer the structure necessary to this research work.

\section{References}

[1] M. Enoki and M. Nagashima, The effect of orbital eccentricity on gravitational wave background radiation from supermassive black hole binaries arXiv:astro-ph/0609377v3 2007

[2] W.A. Hiscock, Low Frequency gravitational waves from black hole macho binaries, Astrophys. J. 509 L101 1998

[3] O.D. Miranda, J.C.N. de Araujo and O.D. Aguiar, Gravitational wave background from stellar black holes in a scenario of structure formation, Class. Quantum Grav. 21 S545 2004

[4] S.W. Hawking and W. Israel, 300 Years of Gravitation, Cambridge University Press, Cambridge 1987.

[5] E.A. Jackson, Equilibrium Statistical Mechanics, Prentice-Hall Inc., Illinois 1968

[6] V. Kalogera, R. O'Shaughnessy and K. Belczynski, Binary compact object coalescence rates: The role of elliptical galaxies arXiv:0908.3635v2 2010

[7] T. Bulik, N. Ivanova, F.A. Rasio, A. Sadowski, K. Belczynski and R. O’Shaughnessy, The total merger rate of compact object binaries in the local universe Astrophys. J. 67611622008

[8] V. Kalogera, K. Belczynski, R. O’Shaughnessy and C. Kim, Constraining population synthesis models via empirical binary compact object merger and supernova rates, Astrophys. J. 6724792008

[9] H.K. Chaurasia and M. Bailes, On the eccentricities and merger rates of double neutron star binaries and the creation of "double supernovae", Astrophys. J. 63210542005

[10] C. R. Evans, I. Ibsen and L. Smarr, Degenerate dwarf binaries as promising, detectable sources of gravitational radiation, Astrophys. J. $\mathbf{3 2 3} 1291987$ 